\title{
Aplicación del Método BATHE en la Valoración Preanestésica Pediátrica Quito-Ecuador, 2016
}

\section{Daniel Renato Reinoso Guadalupe, MD}

Especialista en Anestesiología,

Docente de la Facultad de Medicina, Escuela Superior Politécnica de

Chimborazo Riobamba-Ecuador

Shirley Fernanda Rosero Ordóñez, MD

Magister en Gerencia en Salud para el Desarrollo Local

Docente de la Facultad de Medicina, Escuela Superior Politécnica de

Chimborazo Riobamba-Ecuador

Pedro Renato Flores Brito, MD

Magister en Seguridad y Prevención en Riesgos del Trabajo,

Docente de la Facultad de Medicina, Escuela Superior Politécnica de

Chimborazo Riobamba-Ecuador

Doi: 10.19044/esj.2019.v15n12p469 URL:http://dx.doi.org/10.19044/esj.2019.v15n12p469

\section{Resumen}

El presente estudio se enfoca en el método de BATHE (fondo, afectación, problemas, manejo y empatía) posterior a la entrevista preanestésica, siendo éste un método psicoterapéutico breve que se utiliza para tratar los problemas físicos y psicosociales de los pacientes. La técnica BATHE no ha sido evaluada en el contexto perioperatorio, sino como una forma de mejorar la satisfacción del paciente durante la evaluación preanestésica pediátrica en el Hospital Baca Ortiz durante el período de junio - agosto de 2016. Se utilizaron los métodos observacional, prospectivo y descriptivo, para finalmente aplicar un cuestionario de 20 preguntas basado en el método de BATHE a los padres de los pacientes ASA I y ASA II. La utilización de los métodos antes mencionados arrojó los siguientes resultados: la fiabilidad y confiabilidad del método de BATHE según el instrumento Alpha de Cronbach es del 96,9\% de un total de 150 padres de pacientes encuestados, el grado de satisfacción posterior a la consulta preanestésica fue "alto" en un 48\%; la relación entre la aplicación del método de BATHE y la satisfacción de los padres fue "muy alto" en $50 \%$ y "alto" en un $47,1 \%$. En conclusión, la aplicación del método de BATHE como herramienta en la evaluación preanestésica aumenta la satisfacción de los padres y la confianza del niño que va a ser sometido a cirugía electiva en el Hospital Baca Ortiz de Quito. 
Palabras Clave: pediátrica, satisfacción, paciente, cuestionario

\title{
Application of the BATHE Method in the Pediatric Preanestal Valuation Quito-Ecuador, 2016
}

\author{
Daniel Renato Reinoso Guadalupe, MD \\ Especialista en Anestesiología, \\ Docente de la Facultad de Medicina, Escuela Superior Politécnica de \\ Chimborazo Riobamba-Ecuador \\ Shirley Fernanda Rosero Ordóñez, MD \\ Magister en Gerencia en Salud para el Desarrollo Local \\ Docente de la Facultad de Medicina, Escuela Superior Politécnica de \\ Chimborazo Riobamba-Ecuador \\ Pedro Renato Flores Brito, MD \\ Magister en Seguridad y Prevención en Riesgos del Trabajo, \\ Docente de la Facultad de Medicina, Escuela Superior Politécnica de \\ Chimborazo Riobamba-Ecuador
}

\begin{abstract}
This paper focuses on the use of the BATHE method (background, affectation, problems, management and empathy) after the pre-anesthetic interview. The technique is a brief psychotherapeutic method that is used to treat the physical and psychosocial problems of patients. The BATHE technique has not been evaluated in the perioperative context, but it is considered as a way to improve patient satisfaction during the pre-anesthetic pediatric evaluation at the Baca Ortiz Hospital during June - August 2016. Using the observational, prospective, and descriptive method consists of applying a questionnaire of 20 questions based on the BATHE method to the parents of the ASA I and ASA II patients. The following result was obtained: the reliability of the BATHE method according to the Cronbach Alpha instrument is $96.9 \%$. From a total of 150 parents of patients surveyed, the degree of satisfaction after the pre-anesthetic consultation was "high" by $48 \%$. The relationship between the application of the BATHE Method and the satisfaction of the parents was "very high" at $50 \%$ and "high" at $47.1 \%$. In conclusion, the application of the BATHE method as a tool in the preanesthetic evaluation increases the satisfaction of the parents and the
\end{abstract}


confidence of the child who is going to be subjected to elective surgery in the Baca Ortiz Hospital in Quito.

Keywords: Pediatric, Satisfaction, Patient, Questionnaire

\section{Introduction}

A través de la historia, el hombre se ha inquietado y preocupado por mitigar o al menos controlar el dolor físico (Liondas, 2009). En este sentido, la anestesiología, la cual ha sido considerada como uno de los grandes avances de la humanidad, ha desarrollado a su vez diferentes subespecialidades como: anestesia pediátrica, anestesia cardiovascular, neuroanestesia, anestesia obstétrica, manejo de dolor, entre otras. Esto ha permitido que los anestesiólogos se desempeñen, no solamente en el área de los quirófanos, sino también fuera de los mismos, como médicos perioperatorios que evalúan y tratan a los pacientes de forma exhaustiva en la fase preoperatoria e intraoperatoria manteniendo las constantes vitales óptimas durante la cirugía, y en la fase postoperatoria atendiendo las necesidades analgésicas del paciente (Rivera, 2004).

La anestesiología, como especialidad de la medicina, es la encargada de los aspectos farmacológicos, fisiológicos y clínicos durante el acto quirúrgico, y como todo proceso natural del ser humano a lo desconocido, la misma influye psicológicamente tanto en los padres como en los pacientes que asisten a la consulta preanestésica (Pitarch \& Ibáñez, 2005). Por tal motivo, manejar estos factores y socializarlos con los pacientes y sus padres son determinantes para mejorar los estados de ansiedad y depresión de los mismos; por lo que denota suma importancia el profesionalismo del anestesiólogo en su asistencia médica (Mira \& Aranaz, 2000).

En lo que a esto respecta, es importante conocer métodos y protocolos de manejo para la atención al usuario que acude a la consulta preanestésica, esto con la finalidad de lograr una eficiencia y eficacia de sus funciones y brindar un servicio de calidad al asistido, como método previo a lograr una estabilidad psicología integral que no infiera ni perjudique su estado emocional (Gómez, Vinagre, Hita, \& Macía, 2008). Uno de estos métodos de atención al usuario es el método de BATHE, el cual está basado en una entrevista con preguntas sencillas. Dicha entrevista es aplicable inmediatamente después de la consulta preanestésica y sirve para medir los antecedentes e historial del paciente, empatía, problemas, afectaciones, calidad de manejo hacia el usuario, estado emocional y percepción del servicio recibido, en un entorno familiar y amigable entre el médico anestesiólogo, el paciente y sus padres. Este método resulta ser muy útil pues, dada su breve y fácil naturaleza, puede ser aplicado en corto tiempo ajustándose así a las demandas del servicio, y en lo que respecta a las necesidades del paciente lo 
más importante es que no aumenta el tiempo asignado a la consulta médica (DeMaria, DeMaria, Silvay, \& Flynn, 2011).

\section{Hipótesis}

¿Con la utilización del método BATHE se optimizará la atención alos pacientes que acuden a la consulta preanestésica del Hospital Baca Ortiz en el periodo junio - agosto de 2016 ?

\section{Objetivos}

- Evaluar el grado de satisfacción de los padres de los pacientes, que acuden a la consulta preanestésica pediátrica en el Hospital Baca Ortiz, mediante la aplicación del método de BATHE durante junio - agosto de 2016.

- Identificar los problemas más comunes por los cuales el nivel de satisfacción de los padres de los pacientes que acuden a la consulta preanestésica no es óptimo.

- Mejorar el nivel de seguridad percibido por los padres de los pacientes prequirúrgicos que acuden a la consulta preanestésica pediátrica.

Tabla 1. Definición y operación de la Hipótesis

\begin{tabular}{|c|c|c|c|c|}
\hline VARIABLES & DEFINICIÓN & DIMENSIÓN & INDICADOR & ESCALA \\
\hline Edad & $\begin{array}{l}\text { Tiempo transcurrido desde } \\
\text { su nacimiento hasta la fecha } \\
\text { actual. }\end{array}$ & $\begin{array}{l}\text { Tiempo } \\
\text { trascurrido }\end{array}$ & $\begin{array}{ll}\text { - } & 1 \text { mes a } 2 \text { años } \\
\text { - } & >2 \text { a } 5 \text { años } \\
\text { - } & 6 \text { a } 11 \text { años } \\
\text { - } & 12 \text { a } 15 \text { años. }\end{array}$ & Numérica \\
\hline Sexo & $\begin{array}{l}\text { Características fenotípicas } \\
\text { que diferencian el hombre de } \\
\text { la mujer. }\end{array}$ & Fenotipo & $\begin{array}{l}\text { Hombre } \\
\text { Mujer }\end{array}$ & Nominal \\
\hline Procedencia & $\begin{array}{l}\text { Lugar, cosa o persona del } \\
\text { que procede alguien o algo. }\end{array}$ & Región & $\begin{array}{l}\text { Costa, Sierra, Oriente, } \\
\text { Región Insular }\end{array}$ & Nominal \\
\hline $\begin{array}{l}\text { Estado físico según la } \\
\text { American Society of } \\
\text { Anesthesiologists } \\
\text { Physical Status, (ASA } \\
\text { PS) }\end{array}$ & $\begin{array}{l}\text { Clasificación del estado } \\
\text { físico preoperatorio del } \\
\text { paciente según la Sociedad } \\
\text { Americana de } \\
\text { Anestesiología. }\end{array}$ & $\begin{array}{l}\text { Clasificación } \\
\text { del estado } \\
\text { físico }\end{array}$ & $\begin{array}{l}\text { ASA I } \\
\text { ASA II }\end{array}$ & Ordinal \\
\hline $\begin{array}{l}\text { Satisfacción con } \\
\text { respecto a la consulta } \\
\text { preanestésica }\end{array}$ & $\begin{array}{l}\text { Estado de bienestar existente } \\
\text { entre las expectativas del } \\
\text { paciente y lo que se ha } \\
\text { conseguido con la consulta } \\
\text { preanestésica. }\end{array}$ & $\begin{array}{l}\text { Método de } \\
\text { BATHE }\end{array}$ & $\begin{array}{l}\text { Muy pobre } \\
\text { Pobre } \\
\text { Justa } \\
\text { Buena } \\
\text { Muy buena } \\
\end{array}$ & Ordinal \\
\hline Seguridad & $\begin{array}{l}\text { Sentimiento de protección } \\
\text { frente a carencias y peligros } \\
\text { externos que afecten } \\
\text { negativamente la calidad de } \\
\text { vida. }\end{array}$ & Percepción & $\begin{array}{l}\text { Muy pobre } \\
\text { Pobre } \\
\text { Justa } \\
\text { Buena } \\
\text { Muy buena } \\
\end{array}$ & Ordinal \\
\hline
\end{tabular}

Elaborado por: Equipo de investigación, 2016. 


\section{Tipo de estudio}

El tipo de estudio es "transversal de período", el cual consiste en aplicar un cuestionario de 20 preguntas a los padres de los pacientes que acuden a la consulta preanestésica pediátrica.

\section{Criterios de selección}

Criterios de inclusión:

5) Padres de lactantes y niños de corta edad (de 1 mes a 2 años).

6) Padres de niños, preescolar (de 2 a 5 años).

7) Padres de niños, escolar (de 6 a 11 años).

8) Padres de adolescentes (de 12 a entre 15 años)

9) Padres de pacientes sometidos a cirugía programada

10) Padres de pacientes ASA I y ASA II.

11) Padres de pacientes que aceptan contestar el cuestionario y firman el consentimiento informado.

Criterios de exclusión:

- Padres de pacientes menores de 1 mes y mayores de 16 años de edad.

- Padres de pacientes sometidos a cirugía de emergencia.

- Padres de pacientes ASA III - IV - V - VI.

- Padres de pacientes que no aceptan contestar el cuestionario y que no firman el consentimiento informado.

- Pacientes que acuden a la consulta en compañía de cuidadores y no de sus padres biológicos.

Criterio Éticos:

- Consentimiento informado.

- Declaración de confidencialidad.

\section{Tamaño de la Muestra}

Para el cálculo, se utilizó la fórmula de muestreo simple aleatorio para grupo homogéneo infinito:

$$
\mathrm{n}=\frac{\mathrm{Z}^{2} \mathrm{pq}}{\mathrm{E}^{2}}
$$

Donde

$\boldsymbol{n}=$ equivalente al número de la muestra.

$z=95 \%=1,96$; equivalente al nivel de confianza.

$\boldsymbol{p}=10 \%=(0,1) ;$ equivalente a la variabilidad positiva.

$\boldsymbol{q}=1-p=(0,80)$; equivalente a la variabilidad negativa.

$\boldsymbol{e}=5 \%=0,05^{2}=(0,0025)$; equivalente al error aceptable. 
Se atienden en promedio alrededor de 300 consultas preanestésicas al mes, de las cuales para el mes de enero de 2016 corresponden 248 consultas, para febrero de 2016308 consultas y marzo de 2016315 consultas. Con estos datos, y según la fórmula, nos da una muestra total de 122 padres de pacientes que acuden a la consulta; sin embargo, se realizaron 150 encuestas, equivalentes al $10 \%$ extra de la muestra, por pérdidas y error.

\section{Resultados y discusión}

La aplicación del método BATHE a los padres de los niños que serán intervenidos quirúrgicamente, se presenta en cuatro sesiones. En la primera sesión se realiza el análisis de fiabilidad del instrumento a partir de la determinación del coeficiente Alpha de Cronbach. La segunda sesión está relacionada con el análisis descriptivo de los resultados de cada pregunta. En la tercera sesión se determina la escala de aplicación del método BATHE, atendiendo a los resultados de las cinco primeras preguntas, además de determinar cuál es el grado de satisfacción de los padres de los pacientes considerando los resultados de las preguntas de la 6 a la 20. En la última sesión, se determina de forma estadística la relación que existe entre el grado de aplicación de las variables y el grado de satisfacción de los padres de los pacientes.

\section{Análisis de fiabilidad}

La fiabilidad del instrumento permite garantizar que los resultados obtenidos sean confiables y que las conclusiones a las que se arriba a partir del análisis de los mismos tengan un sustento válido. El instrumento utilizado para determinar la fiabilidad es el coeficiente Alpha de Cronbach.

Tabla 2. Coeficiente Alpha de Cronbach

\begin{tabular}{|c|c|c|}
\hline $\begin{array}{c}\text { ALFA DE } \\
\text { CRONBACH }\end{array}$ & $\begin{array}{c}\text { ALFA DE CRONBACH BASADA EN LOS } \\
\text { ELEMENTOS TIPIFICADOS }\end{array}$ & $\begin{array}{c}\text { NÚMERO DE } \\
\text { ELEMENTOS }\end{array}$ \\
\hline 0,969 & 0,971 & 15 \\
\hline
\end{tabular}

Elaborado por: Equipo de investigación,2016.

El coeficiente Alpha de Cronbach, es del 96,9\%, lo que representa un alto nivel de confiabilidad.

\section{Análisis descriptivo}

Se realiza a partir de la elaboración de tablas, que están incluidas en el método BATHE. 
Tabla 3. ¿El médico le preguntó a usted, cuál es el motivo de su visita o que lo trae por aquí hoy?

\begin{tabular}{|c|c|c|c|c|c|}
\hline & & Frecuencia & Porcentaje & $\begin{array}{c}\text { Porcentaje } \\
\text { válido }\end{array}$ & $\begin{array}{c}\text { Porcentaje } \\
\text { acumulado }\end{array}$ \\
\hline \multirow{3}{*}{ Válidos } & Sí & 54 & 36,0 & 36,0 & 36,0 \\
\cline { 2 - 6 } & No & 96 & 64,0 & 64,0 & 100,0 \\
\cline { 2 - 6 } & Total & 150 & 100,0 & 100,0 & \\
\hline
\end{tabular}

Elaborado por: Equipo de investigación, 2016.

En la pregunta 1 (tabla 3), el 54\% de los encuestados afirma que el médico le pregunta acerca del motivo de la visita al hospital, esto representa el 36\%. El $64 \%$ restante, responde que el médico no se ha interesado sobre el motivo de la visita al hospital. Este comportamiento resulta desfavorable en el grado de aplicación final del método BATHE.

Tabla 4. ¿El médico le preguntó a usted, cómo le está afectando la idea de operar a su hijo/a?

\begin{tabular}{|c|c|c|c|c|c|}
\hline & & Frecuencia & Porcentaje & $\begin{array}{c}\text { Porcentaje } \\
\text { válido }\end{array}$ & $\begin{array}{c}\text { Porcentaje } \\
\text { acumulado }\end{array}$ \\
\hline \multirow{3}{*}{ Válidos } & Sí & 46 & 30,7 & 30,7 & 30,7 \\
\cline { 2 - 6 } & No & 104 & 69,3 & 69,3 & 100,0 \\
\cline { 2 - 6 } & Total & 150 & 100,0 & 100,0 & \\
\hline
\end{tabular}

Elaborado por: Equipo de investigación, 2016.

En la pregunta 2 (tabla 4), con relación a si el médico les ha preguntado a los padres sobre cómo les afecta la idea de operar a su hijo, solo $46 \%$ de los encuestados ha dado una respuesta afirmativa, que representa el 30,7 \%; mientras que el 69,3\% responde que el médico no le ha realizado la pregunta. Estas respuestas influyen de forma negativa en el grado de aplicación del método BATHE.

Tabla 5. ¿El médico le preguntó a usted, cuáles son sus temores respecto a la cirugía?

\begin{tabular}{|c|c|c|c|c|c|}
\hline & Frecuencia & Porcentaje & $\begin{array}{c}\text { Porcentaje } \\
\text { válido }\end{array}$ & $\begin{array}{c}\text { Porcentaje } \\
\text { acumulado }\end{array}$ \\
\hline \multirow{3}{*}{ Válidos } & Sí & 112 & 74,7 & 74,7 & 74,7 \\
\cline { 2 - 6 } & No & 38 & 25,3 & 25,3 & 100,0 \\
\cline { 2 - 6 } & Total & 150 & 100,0 & 100,0 & \\
\hline
\end{tabular}

Elaborado por: Equipo de investigación, 2016.

A la pregunta 3 (tabla 5), que trata sobre si el médico les preguntó sobre los temores relacionados con la cirugía de su hijo 112 padres respondieron de manera afirmativa, lo que representa el 74,7 \% de los encuestados; sólo el 25,3 $\%$ respondió de forma negativa. Las respuestas en esta pregunta ponen de manifiesto un alto grado de aplicación del método BATHE. 
Tabla 6. ¿El médico le preguntó a usted, cómo está manejando estas preocupaciones?

\begin{tabular}{|c|c|c|c|c|c|}
\hline & & Frecuencia & Porcentaje & $\begin{array}{c}\text { Porcentaje } \\
\text { válido }\end{array}$ & $\begin{array}{c}\text { Porcentaje } \\
\text { acumulado }\end{array}$ \\
\hline \multirow{3}{*}{ Válidos } & Sí & 63 & 42,0 & 42,0 & 42,0 \\
\cline { 2 - 6 } & No & 87 & 58,0 & 58,0 & 100,0 \\
\cline { 2 - 6 } & Total & 150 & 100,0 & 100,0 & \\
\hline
\end{tabular}

Elaborado por: Equipo de investigación, 2016.

A la pregunta 4 (tabla 6), que trata sobre si el médico les preguntó a los padres de los pacientes acerca de cómo están manejando estas preocupaciones, 63 personas responden que el médico sí les hizo una pregunta relacionada con el manejo de sus preocupaciones; esto representa un $42 \%$, mientras que el resto de los padres que representan el $58 \%$ dicen que el médico no hizo alusión a esta pregunta. La influencia sobre el grado de utilización del método BATHE es entonces negativa.

Tabla 7. ¿El médico, fue comprensivo con sus preocupaciones?

\begin{tabular}{|c|c|c|c|c|c|}
\hline & & Frecuencia & Porcentaje & $\begin{array}{c}\text { Porcentaje } \\
\text { válido }\end{array}$ & $\begin{array}{c}\text { Porcentaje } \\
\text { acumulado }\end{array}$ \\
\hline \multirow{3}{*}{ Válidos } & Sí & 123 & 82,0 & 82,0 & 82,0 \\
\cline { 2 - 6 } & No & 27 & 18,0 & 18,0 & 100,0 \\
\cline { 2 - 6 } & Total & 150 & 100,0 & 100,0 & \\
\hline
\end{tabular}

Elaborado por: Equipo de investigación, 2016.

En la pregunta 5 (tabla 7), acerca del nivel de comprensión de los médicos sobre las preocupaciones de los padres de los pacientes, 123 de los 150 padres responden que los médicos sí se mostraron comprensibles con las preocupaciones acerca del nivel de preocupaciones por la operación de sus hijos. Los 27 padres restantes que representan el $18 \%$ afirman que los médicos no se mostraron compresivos sobre sus preocupaciones. En esta pregunta se muestra un alto grado de cumplimiento con el método BATHE.

Tabla 8. Amabilidad de su médico

\begin{tabular}{|c|c|c|c|c|c|}
\hline \multicolumn{2}{|c|}{} & Frecuencia & Porcentaje & $\begin{array}{c}\text { Porcentaje } \\
\text { válido }\end{array}$ & $\begin{array}{c}\text { Porcentaje } \\
\text { acumulado }\end{array}$ \\
\hline \multirow{7}{*}{ Válidos } & Muy pobre & 1 & 0,7 & 0,7 & 0,7 \\
\cline { 2 - 6 } & Pobre & 4 & 2,7 & 2,7 & 3,3 \\
\cline { 2 - 6 } & Justa & 18 & 12,0 & 12,0 & 15,3 \\
\cline { 2 - 6 } & Buena & 94 & 62,7 & 62,7 & 78,0 \\
\cline { 2 - 6 } & Muy buena & 33 & 22,0 & 22,0 & 100,0 \\
\cline { 2 - 6 } & Total & 150 & 100,0 & 100,0 & \\
\hline
\end{tabular}

Elaborado por: Equipo de investigación, 2016.

En la pregunta 6 (tabla 8), las respuestas se clasificaron según las siguientes categorías:

- Muy pobre

- Pobre

- Justa 
- Buena

- Muy Buena

El $62,7 \%$ de los padres encuestados considera como buena la amabilidad de los médicos y el $22 \%$ lo considera muy buena. De forma general el $84,7 \%$ da una evaluación satisfactoria respecto a la amabilidad de los médicos. Solo el 15,3\% considera estos aspectos entre muy pobre y justo. Las respuestas dadas por los padres de los pacientes se consideran favorables, teniendo en cuenta el grado de satisfacción de los mismos.

Tabla 9. Explicación del médico sobre las preguntas que usted tuvo

\begin{tabular}{|c|c|c|c|c|c|}
\hline \multicolumn{2}{|c|}{} & Frecuencia & Porcentaje & $\begin{array}{c}\text { Porcentaje } \\
\text { válido }\end{array}$ & $\begin{array}{c}\text { Porcentaje } \\
\text { acumulado }\end{array}$ \\
\hline \multirow{4}{*}{ Válidos } & Muy pobre & 1 & 0,7 & 0,7 & 0,7 \\
\cline { 2 - 6 } & Pobre & 8 & 5,3 & 5,3 & 6,0 \\
\cline { 2 - 6 } & Justa & 17 & 11,3 & 11,3 & 17,3 \\
\cline { 2 - 6 } & Buena & 90 & 60,0 & 60,0 & 77,3 \\
\cline { 2 - 6 } & Muy buena & 34 & 22,7 & 22,7 & 100,0 \\
\cline { 2 - 6 } & Total & 150 & 100,0 & 100,0 & \\
\hline
\end{tabular}

Elaborado por: Equipo de investigación, 2016.

La pregunta 7 (tabla 9), está relacionada con la explicación de los médicos sobre las preguntas que hicieron los padres. El $60 \%$ de los encuestados responde que considera como buenas las explicaciones de los médicos y un $22,7 \%$ las califican como muy buenas. Sólo un $12,3 \%$ califica entre uy pobre y justa la respuesta de los médicos a las preguntas de los padres. Este resultado se presenta como positivo al grado de satisfacción de los padres de los pacientes.

Tabla 10. Preocupación del médico sobre sus miedos

\begin{tabular}{|c|c|c|c|c|c|}
\hline \multicolumn{2}{|c|}{} & Frecuencia & Porcentaje & $\begin{array}{c}\text { Porcentaje } \\
\text { válido }\end{array}$ & $\begin{array}{c}\text { Porcentaje } \\
\text { acumulado }\end{array}$ \\
\hline Válidos & Muy pobre & 1 & 0,7 & 0,7 & 0,7 \\
\cline { 2 - 6 } & Pobre & 15 & 10,0 & 10,0 & 10,7 \\
\cline { 2 - 6 } & Justa & 25 & 16,7 & 16,7 & 27,3 \\
\cline { 2 - 6 } & Buena & 85 & 56,7 & 56,7 & 84,0 \\
\cline { 2 - 6 } & Muy buena & 24 & 16,0 & 16,0 & 100,0 \\
\cline { 2 - 6 } & Total & 150 & 100,0 & 100,0 & \\
\hline
\end{tabular}

Elaborado por: Equipo de investigación, 2016.

La pregunta 8 (tabla 10), está relacionada con la preocupación de los médicos sobre los miedos de los padres de los niños que serán intervenidos quirúrgicamente. El $56,7 \%$ de los padres encuestados considera que la preocupación de los médicos sobre sus miedos es buena y un $16 \%$ lo califica de muy bueno. Un 27,4\% piensan que la preocupación de los médicos se puede calificar entre muy pobre y justa. De forma general estos resultados se visualizan como positivos para la satisfacción de los padres. 
Tabla 11. Esfuerzo del médico por incluirle en las decisiones sobre la anestesia

\begin{tabular}{|c|c|c|c|c|c|}
\hline \multicolumn{2}{|c|}{} & Frecuencia & Porcentaje & $\begin{array}{c}\text { Porcentaj } \\
\text { e válido }\end{array}$ & $\begin{array}{c}\text { Porcentaje } \\
\text { acumulado }\end{array}$ \\
\hline Válidos & Muy pobre & 12 & 8,0 & 8,0 & 8,0 \\
\cline { 2 - 6 } & Pobre & 18 & 12,0 & 12,0 & 20,0 \\
\cline { 2 - 6 } & Justa & 28 & 18,7 & 18,7 & 38,7 \\
\cline { 2 - 6 } & Buena & 64 & 42,7 & 42,7 & 81,3 \\
\cline { 2 - 6 } & Muy buena & 28 & 18,7 & 18,7 & 100,0 \\
\cline { 2 - 6 } & Total & 150 & 100,0 & 100,0 & \\
\hline
\end{tabular}

Elaborado por: Equipo de investigación, 2016.

En la pregunta 9 (tabla 11), el 42,7\% considera como bueno el esfuerzo de los médicos hacia los padres de los niños que serán operados, para que tengan algún tipo de decisión en cuanto a la anestesia que se aplicará durante la operación; el $18,7 \%$ de los padres lo califican como muy bueno. Sin embargo, en este aspecto un porcentaje considerable lo califica como pobre y muy pobre, exactamente un $20 \%$.

Tabla 12. Información sobre el tipo de anestesia

\begin{tabular}{|c|c|c|c|c|c|}
\hline \multicolumn{2}{|c|}{} & Frecuencia & Porcentaje & $\begin{array}{c}\text { Porcentaje } \\
\text { válido }\end{array}$ & $\begin{array}{c}\text { Porcentaje } \\
\text { acumulado }\end{array}$ \\
\hline \multirow{4}{*}{ Válidos } & Muy pobre & 1 & 0,7 & 0,7 & 0,7 \\
\cline { 2 - 6 } & Pobre & 3 & 2,0 & 2,0 & 2,7 \\
\cline { 2 - 6 } & Justa & 20 & 13,3 & 13,3 & 16,0 \\
\cline { 2 - 6 } & Buena & 90 & 60,0 & 60,0 & 76,0 \\
\cline { 2 - 6 } & Muy buena & 36 & 24,0 & 24,0 & 100,0 \\
\cline { 2 - 6 } & Total & 150 & 100,0 & 100,0 & \\
\hline
\end{tabular}

Elaborado por: Equipo de investigación, 2016.

La pregunta 10 (tabla 12), está relacionada con la información que brindan los médicos sobre la anestesia. E1 60\% de los padres califica este punto como bueno, mientras que un $24 \%$ dice que la información brindada es muy buena. Sólo un $16 \%$ de los padres encuestados dice que la información que brindan los médicos sobre la anestesia es entre muy pobre y justa.

Tabla 13. Instrucciones sobre el seguimiento y recuperación de la anestesia

\begin{tabular}{|c|c|c|c|c|c|}
\hline \multicolumn{2}{|c|}{} & Frecuencia & Porcentaje & $\begin{array}{c}\text { Porcentaje } \\
\text { válido }\end{array}$ & $\begin{array}{c}\text { Porcentaje } \\
\text { acumulado }\end{array}$ \\
\hline \multirow{4}{*}{ Válidos } & Muy pobre & 4 & 2,7 & 2,7 & 2,7 \\
\cline { 2 - 6 } & Pobre & 10 & 6,7 & 6,7 & 9,3 \\
\cline { 2 - 6 } & Justa & 28 & 18,7 & 18,7 & 28,0 \\
\cline { 2 - 6 } & Buena & 77 & 51,3 & 51,3 & 79,3 \\
\cline { 2 - 6 } & Muy buena & 31 & 20,7 & 20,7 & 100,0 \\
\cline { 2 - 6 } & Total & 150 & 100,0 & 100,0 & \\
\hline
\end{tabular}

Elaborado por: Equipo de investigación, 2016.

La pregunta 11 (tabla 13), arroja valores del2.6 \% y $6.6 \%$ de los pacientes, quienes opinan que es "muy pobre" y "pobre" respectivamente. 
Otro $18.67 \%$ de los pacientes opinan que es "justa", un 51.33\% la valora como "buena" y el 20.67\% restante como "muy buena". Estoimplica una incidencia positiva en el índice de satisfacción de los pacientes, ya que más de la mitad de los encuestados quedaron satisfechos.

Tabla 14. Uso de palabras comprensibles por parte del médico

\begin{tabular}{|c|c|c|c|c|c|}
\hline \multicolumn{2}{|c|}{} & Frecuencia & Porcentaje & $\begin{array}{c}\text { Porcentaje } \\
\text { válido }\end{array}$ & $\begin{array}{c}\text { Porcentaje } \\
\text { acumulado }\end{array}$ \\
\hline \multirow{4}{*}{ Válidos } & Muy pobre & 3 & 2,0 & 2,0 & 2,0 \\
\cline { 2 - 6 } & Pobre & 5 & 3,3 & 3,3 & 5,3 \\
\cline { 2 - 6 } & Justa & 25 & 16,7 & 16,7 & 22,0 \\
\cline { 2 - 6 } & Buena & 80 & 53,3 & 53,3 & 75,3 \\
\cline { 2 - 6 } & Muy buena & 37 & 24,7 & 24,7 & 100,0 \\
\cline { 2 - 6 } & Total & 150 & 100,0 & 100,0 & \\
\hline
\end{tabular}

Elaborado por: Equipo de investigación, 2016.

En la pregunta 12 (tabla 14), sobre el uso de palabras comprensibles por parte del médico, sólo el $2 \%$ y $3.3 \%$ de los pacientes opinó que el uso de palabras comprensibles por parte del médico fue "muy pobre" y "pobre" respectivamente. Sin embargo, la mayoría de los encuestados refirieron una comprensión "justa", "buena" y "muy buena" del lenguaje utilizado por el médico, con valores de $16.67 \%, 53.33 \%$ y $24.67 \%$ respectivamente. Estos resultados indican que la mayoría de los pacientes encuestados quedaron satisfechos con su interacción con el médico, aumentando así el índice de satisfacción.

Tabla 15. Cantidad de tiempo que el médico pasó con usted

\begin{tabular}{|l|c|c|c|c|c|}
\hline \multirow{4}{*}{} & Frecuencia & Porcentaje & $\begin{array}{c}\text { Porcentaje } \\
\text { válido }\end{array}$ & $\begin{array}{c}\text { Porcentaje } \\
\text { acumulado }\end{array}$ \\
\hline \multirow{4}{*}{ Válidos } & Muy pobre & 18 & 12,0 & 12,0 & 12,0 \\
\cline { 2 - 6 } & Pobre & 24 & 16,0 & 16,0 & 28,0 \\
\cline { 2 - 6 } & Justa & 34 & 22,7 & 22,7 & 50,7 \\
\cline { 2 - 6 } & Buena & 55 & 36,7 & 36,7 & 87,3 \\
\cline { 2 - 6 } & Muy buena & 19 & 12,7 & 12,7 & 100,0 \\
\cline { 2 - 6 } & Total & 150 & 100,0 & 100,0 & \\
\hline
\end{tabular}

Elaborado por: Equipo de investigación, 2016

En la pregunta 13 (tabla 15), con respecto a la cantidad de tiempo que el médico dedicó a la consulta hay opiniones divididas. El 12\% la valoran como "muy pobre", el 16\% como "pobre", el 22.67\% como "justa", el 36.67\% como "buena" y el $12.67 \%$ restante como "muy buena". Esto da como resultado que, poco más de la mitad de los padres de los pacientes opina que el médico no pasó un tiempo adecuado con ellos, lo cual incide negativamente en el índice de satisfacción de la consulta preanestésica. 
Tabla 16. Nivel de confianza sobre el médico que les atendió

\begin{tabular}{|c|c|c|c|c|c|}
\hline & Frecuencia & Porcentaje & $\begin{array}{c}\text { Porcentaje } \\
\text { válido }\end{array}$ & $\begin{array}{c}\text { Porcentaje } \\
\text { acumulado }\end{array}$ \\
\hline \multirow{4}{*}{ Válidos } & Muy pobre & 4 & 2,7 & 2,7 & 2,7 \\
\cline { 2 - 6 } & Pobre & 8 & 5,3 & 5,3 & 8,0 \\
\cline { 2 - 6 } & Justa & 25 & 16,7 & 16,7 & 24,7 \\
\cline { 2 - 6 } & Buena & 83 & 55,3 & 55,3 & 80,0 \\
\cline { 2 - 6 } & Muy buena & 30 & 20,0 & 20,0 & 100,0 \\
\cline { 2 - 6 } & Total & 150 & 100,0 & 100,0 & \\
\hline
\end{tabular}

Elaborado por: Equipo de investigación, 2016.

En la pregunta 14 (tabla 16), se puede observar que el nivel de confianza de los encuestados, sobre el médico que les atendió es mayoritariamente bueno. Sólo un $2.7 \%$ lo cataloga como "muy pobre", un $5.333 \%$ como "pobre" y un $16.67 \%$ como "justo". El porcentaje restante de los encuestados opinan positivamente, un $53.33 \%$ de ellos como "bueno" y un $20 \%$ como "muy bueno", lo cual eleva el índice de satisfacción de la consulta.

Tabla 17. Recomendación del médico para otras personas

\begin{tabular}{|c|c|c|c|c|c|}
\hline \multicolumn{2}{|c|}{} & Frecuencia & Porcentaje & $\begin{array}{c}\text { Porcentaje } \\
\text { válido }\end{array}$ & $\begin{array}{c}\text { Porcentaje } \\
\text { acumulado }\end{array}$ \\
\hline \multirow{4}{*}{ Válidos } & Muy pobre & 2 & 1,3 & 1,3 & 1,3 \\
\cline { 2 - 6 } & Pobre & 11 & 7,3 & 7,3 & 8,7 \\
\cline { 2 - 6 } & Justa & 28 & 18,7 & 18,7 & 27,3 \\
\cline { 2 - 6 } & Buena & 83 & 55,3 & 55,3 & 82,7 \\
\cline { 2 - 6 } & Muy buena & 26 & 17,3 & 17,3 & 100,0 \\
\cline { 2 - 6 } & Total & 150 & 100,0 & 100,0 & \\
\hline
\end{tabular}

Elaborado por: Equipo de investigación, 2016.

En la pregunta 15 (tabla 17), en relación a la recomendación del médico con otras personas, el 1.3\% de los encuestados opinó "muy pobre", el 7.333\% "pobre", el 18.67\% "justa", el 55.33\% "buena" y el restante 17.33\% "muy buena". Esto indica que la mayoría de ellos recomendaría su médico a otras personas, influyendo de forma positiva en el índice de satisfacción de los padres de los pacientes.

Tabla 18. Percepción del cuidado que recibirá por parte del anestesiólogo

\begin{tabular}{|c|c|c|c|c|c|}
\hline \multicolumn{2}{|c|}{} & Frecuencia & Porcentaje & $\begin{array}{c}\text { Porcentaje } \\
\text { válido }\end{array}$ & $\begin{array}{c}\text { Porcentaje } \\
\text { acumulado }\end{array}$ \\
\hline \multirow{4}{*}{ Válidos } & Pobre & 9 & 6,0 & 6,0 & 6,0 \\
\cline { 2 - 6 } & Justa & 16 & 10,7 & 10,7 & 16,7 \\
\cline { 2 - 6 } & Buena & 89 & 59,3 & 59,3 & 76,0 \\
\cline { 2 - 6 } & Muy buena & 36 & 24,0 & 24,0 & 100,0 \\
\cline { 2 - 6 } & Total & 150 & 100,0 & 100,0 & \\
\hline
\end{tabular}

Elaborado por: Equipo de investigación, 2016.

La respuesta a la pregunta 16 (tabla 18), sobre la percepción del cuidado que recibirán por parte del anestesiólogo es muy positiva ya que, 
solamente un 6\% opinó "pobre" y un 10.67\% "justa"; sin embargo, el 59.33\% la catalogaron como "buena" y el $24 \%$ como "muy buena", lo que incrementa el índice de satisfacción por la consulta.

Tabla 19. ¿Cree que su hijo/a está preparado para la recuperación después de la cirugía, basado en la consulta preanestésica?

\begin{tabular}{|c|c|c|c|c|c|}
\hline \multicolumn{2}{|c|}{} & Frecuencia & Porcentaje & $\begin{array}{c}\text { Porcentaje } \\
\text { válido }\end{array}$ & $\begin{array}{c}\text { Porcentaje } \\
\text { acumulado }\end{array}$ \\
\hline \multirow{4}{*}{ Válidos } & Muy pobre & 1 & 0,7 & 0,7 & 0,7 \\
\cline { 2 - 6 } & Pobre & 5 & 3,3 & 3,3 & 4,0 \\
\cline { 2 - 6 } & Justa & 22 & 14,7 & 14,7 & 18,7 \\
\cline { 2 - 6 } & Buena & 93 & 62,0 & 62,0 & 80,7 \\
\cline { 2 - 6 } & Muy buena & 29 & 19,3 & 19,3 & 100,0 \\
\cline { 2 - 6 } & Total & 150 & 100,0 & 100,0 & \\
\hline
\end{tabular}

Elaborado por: Equipo de investigación, 2016.

En la pregunta 17 (tabla 19), un gran porcentaje de encuestados cree que su hijo/a está preparado para la recuperación después de la cirugía, basado en la consulta preanestésica. Únicamente el $0.7 \%$ respondió "muy pobre", un $3.333 \%$ "pobre" y un 14.67\% "justa". El 62\% opinó "buena" y el $19.33 \%$ "muy buena", valores que confirman un resultado positivo en el índice de satisfacción de los padres.

Tabla 20. Opinión personal del médico que les atendió

\begin{tabular}{|c|c|c|c|c|c|}
\hline \multicolumn{2}{|c|}{} & Frecuencia & Porcentaje & $\begin{array}{c}\text { Porcentaje } \\
\text { válido }\end{array}$ & $\begin{array}{c}\text { Porcentaje } \\
\text { acumulado }\end{array}$ \\
\hline \multirow{4}{*}{ Válidos } & Muy pobre & 1 & 0,7 & 0,7 & 0,7 \\
\cline { 2 - 6 } & Pobre & 8 & 5,3 & 5,3 & 6,0 \\
\cline { 2 - 6 } & Justa & 16 & 10,7 & 10,7 & 16,7 \\
\cline { 2 - 6 } & Buena & 87 & 58,0 & 58,0 & 74,7 \\
\cline { 2 - 6 } & Muy buena & 38 & 25,3 & 25,3 & 100,0 \\
\cline { 2 - 6 } & Total & 150 & 100,0 & 100,0 & \\
\hline
\end{tabular}

Elaborado por: Equipo de investigación, 2016.

En cuanto a la pregunta 18 (tabla 20), muy positiva es la opinión personal de los padres de los pacientes sobre el médico que les atendió, ya que el $25.33 \%$ y $58 \%$ tienen una opinión como "muy buena" y "buena" respectivamente, dejando sólo un 10.67\% que opinó "justa", un 5.3\% "pobre" y un $0.7 \%$ "muy pobre", lo cual hace que el índice de satisfacción por la consulta, se incremente. 
Tabla 21. ¿Qué tanto alivio le brindó la consulta preanestésica, sobre sus dudas y la ansiedad por la cirugía?

\begin{tabular}{|c|c|c|c|c|c|}
\hline \multicolumn{2}{|c|}{} & Frecuencia & Porcentaje & $\begin{array}{c}\text { Porcentaje } \\
\text { válido }\end{array}$ & $\begin{array}{c}\text { Porcentaje } \\
\text { acumulado }\end{array}$ \\
\hline \multirow{4}{*}{ Válidos } & Muy pobre & 3 & 2,0 & 2,0 & 2,0 \\
\cline { 2 - 6 } & Pobre & 7 & 4,7 & 4,7 & 6,7 \\
\cline { 2 - 6 } & Justa & 19 & 12,7 & 12,7 & 19,3 \\
\cline { 2 - 6 } & Buena & 86 & 57,3 & 57,3 & 76,7 \\
\cline { 2 - 6 } & Muy buena & 35 & 23,3 & 23,3 & 100,0 \\
\cline { 2 - 6 } & Total & 150 & 100,0 & 100,0 & \\
\hline
\end{tabular}

Elaborado por: Equipo de investigación, 2016.

En la pregunta 19 (tabla 21), con respecto al alivio brindado por la consulta en cuanto a las dudas y ansiedad por la cirugía, del total de encuestados un $23.33 \%$, un $57.33 \%$ y un $12.67 \%$ lo catalogan como "muy bueno", "bueno" y "justo" respectivamente. Los restantes $4.667 \%$ como "pobre" y 2\% como "muy pobre". Estos valores evidencian la satisfacción de los padres de los pacientes en este aspecto y hacen aumentar el indicador general.

Tabla 22. Satisfacción general posterior a la consulta preanestésica

\begin{tabular}{|c|c|c|c|c|c|}
\hline \multicolumn{2}{|c|}{} & Frecuencia & Porcentaje & $\begin{array}{c}\text { Porcentaj } \\
\text { e válido }\end{array}$ & $\begin{array}{c}\text { Porcentaje } \\
\text { acumulado }\end{array}$ \\
\hline \multirow{4}{*}{ Válidos } & Muy pobre & 4 & 2,7 & 2,7 & 2,7 \\
\cline { 2 - 6 } & Pobre & 6 & 4,0 & 4,0 & 6,7 \\
\cline { 2 - 6 } & Justa & 17 & 11,3 & 11,3 & 18,0 \\
\cline { 2 - 6 } & Buena & 86 & 57,3 & 57,3 & 75,3 \\
\cline { 2 - 6 } & Muy buena & 37 & 24,7 & 24,7 & 100,0 \\
\cline { 2 - 6 } & Total & 150 & 100,0 & 100,0 & \\
\hline
\end{tabular}

Elaborado por: Equipo de investigación, 2016.

Para la pregunta 20 (tabla 22), "muy buena" y "buena" fueron las respuestas del $24.67 \%$ y $57.33 \%$ de los encuestados, respectivamente, sobre su satisfacción general posterior a la consulta preanestésica, dejando un $11.33 \%$ cuya satisfacción fue "justa", un $4 \%$ "pobre" y un $2.667 \%$ "muy pobre". Estos resultados indican un alto grado de complacencia en dicha consulta de manera general, por lo tanto, un incremento en los índices generales de satisfacción de los padres de los pacientes.

\section{Grado de aplicación del MÉTODO DE BATHE}

En este apartado se determina el grado de aplicación del método de BATHE por los médicos durante la presente investigación. Para ello se utilizan las respuestas a las cinco preguntas del cuestionario. Las respuestas positivas son calificadas como 1 y las respuestas negativas se califican como 0 , a partir de ello se establecen las siguientes categorías:

- Nulo: 0 
- Muy bajo: 1

- Bajo: 2

- Medio: 3

- Alto: 4

- Muy alto: 5

Tabla 23. Grado de aplicación del método de BATHE

\begin{tabular}{|c|c|c|c|c|c|}
\hline \multicolumn{2}{|c|}{} & Frecuencia & Porcentaje & $\begin{array}{c}\text { Porcentaje } \\
\text { válido }\end{array}$ & $\begin{array}{c}\text { Porcentaje } \\
\text { acumulado }\end{array}$ \\
\hline \multirow{4}{*}{ Válidos } & Nulo & 14 & 9.3 & 9.3 & 9.3 \\
\cline { 2 - 6 } & Muy bajo & 15 & 10.0 & 10.0 & 19.3 \\
\cline { 2 - 6 } & Bajo & 57 & 38.0 & 38.0 & 57.3 \\
\cline { 2 - 6 } & Medio & 21 & 14.0 & 14.0 & 71.3 \\
\cline { 2 - 6 } & Alto & 9 & 6.0 & 6.0 & 77.3 \\
\cline { 2 - 6 } & Muy alto & 34 & 22.7 & 22.7 & 100.0 \\
\cline { 2 - 6 } & Total & 150 & 100.0 & 100.0 & \\
\hline
\end{tabular}

Elaborado por: Equipo de investigación, 2016.

La tabla 23 muestra los resultados del grado de aplicación del método BATHE por cada padre de paciente encuestado. Los resultados muestran que en el $38 \%$ de los casos el grado de aplicación del método antes mencionado es bajo, el $10 \%$ es muy bajo y en un 9,3\% de los casos es nulo; de forma general, en el 57,3\% de los casos el grado de aplicación de este método no cumple con las expectativas. En el 22,7\% de los casos el grado de aplicación del método es alto, el $14 \%$ es medio y sólo un $6 \%$ corresponde a un grado alto de aplicación.

\section{Grado de satisfacción de los padres}

En este apartado se determina, el grado de satisfacción de los padres con la atención en la consulta preanestésica de sus hijos. Para determinar el grado de satisfacción se utilizó la siguiente escala con el objetivo de dar una valoración objetiva:

- Muy bajo: 0-15 puntos

- Bajo: 16- 30 puntos

- Regular: 31-45 puntos

- Alto: 46-60 puntos

- Muy alto: 61-75 puntos 
Tabla 24. Grado de satisfacción de los padres

\begin{tabular}{|c|c|c|c|c|c|}
\hline \multicolumn{2}{|c|}{} & Frecuencia & Porcentaje & $\begin{array}{c}\text { Porcentaje } \\
\text { válido }\end{array}$ & $\begin{array}{c}\text { Porcentaje } \\
\text { acumulado }\end{array}$ \\
\hline \multirow{4}{*}{ Válidos } & Bajo & 6 & 4.0 & 4.0 & 4.0 \\
\cline { 2 - 6 } & Regular & 13 & 8.7 & 8.7 & 12.7 \\
\cline { 2 - 6 } & Alto & 72 & 48.0 & 48.0 & 60.7 \\
\cline { 2 - 6 } & Muy alto & 59 & 39.3 & 39.3 & 100.0 \\
\cline { 2 - 6 } & Total & 150 & 100.0 & 100.0 & \\
\hline
\end{tabular}

Elaborado por: Equipo de investigación, 2016.

En la tabla 24 se presenta la distribución porcentual y de frecuencia del grado de satisfacción de los padres con la atención en la consulta preoperatoria de sus hijos. En su mayoría que representa el $48 \%$ de los padres encuestados el nivel de satisfacción es alto y un 39,3\% tiene un nivel de satisfacción muy alto. Tan solo un 12,67 \% de los padres encuestados tiene nivel de satisfacción entre regular y bajo.

Tabla 25. Grado de aplicación del MÉTODO de BATHE / Grado de satisfacción de los padres

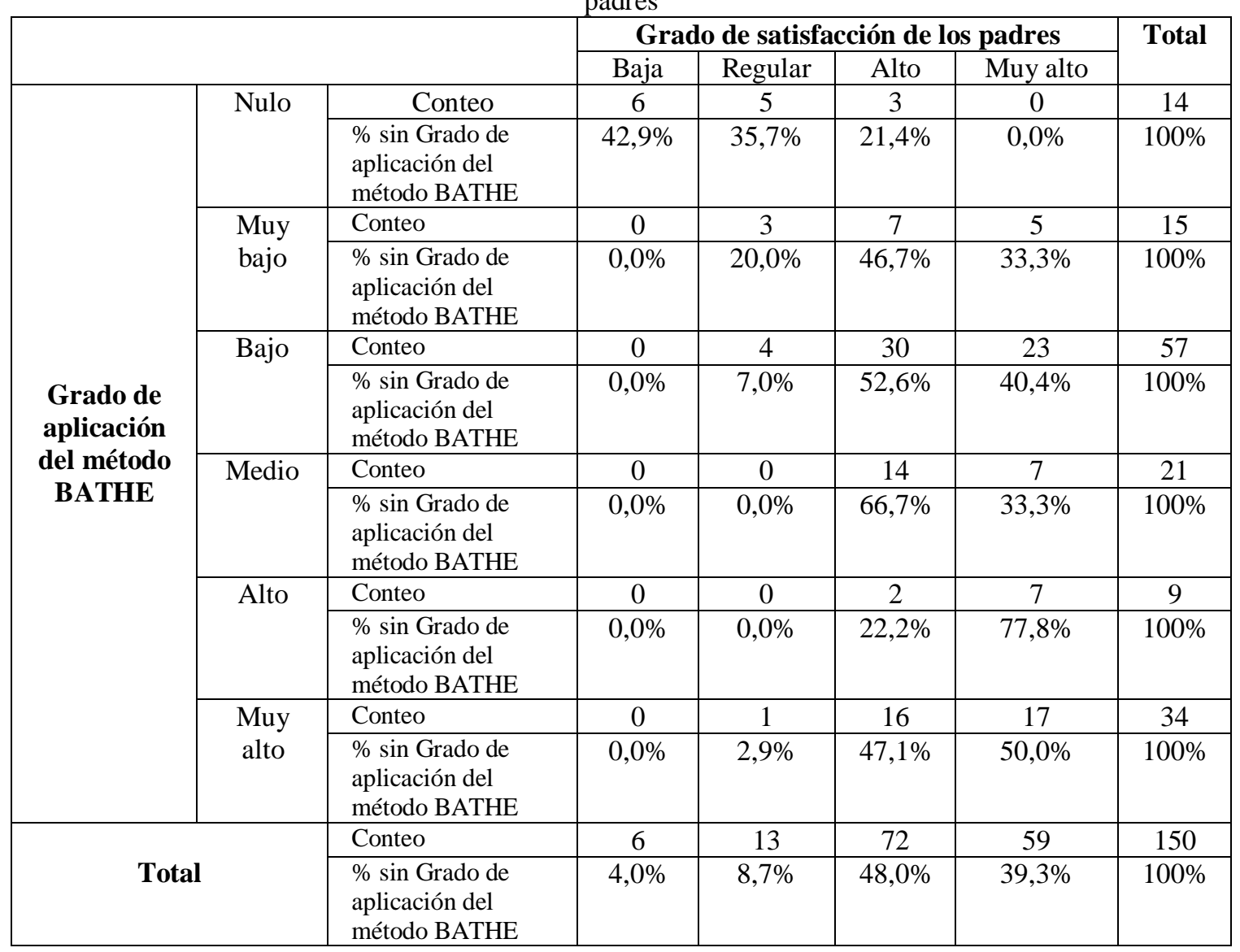

Elaborado por: Equipo de investigación, 2016. 
Para determinar la relación existente entre la utilización del método de BATHE y la satisfacción de los padres, se procede a elaborar la tabla 25 de contingencia entre las variables. El análisis de contingencia presentado muestra que de los casos cuyo grado de aplicación del método es muy alto, la satisfacción de los padres presenta un 50\% muy alta y un $47.1 \%$ alta; es decir, prácticamente en su totalidad presentan resultados satisfactorios. De los casos donde el grado de aplicación del método es alto, el 78,8 \% de los padres tienen un grado muy alto de satisfacción y el 22,2 \% restante tiene un nivel alto de satisfacción. Este comportamiento que se presenta en la tabla de contingencia de las variables puede marcar una tendencia evidenciando una posible relación entre las mismas. Para corroborarlo, se procede a la determinación del estadístico Chi-Cuadrado de Pearson (tabla 26).

Tabla 26. Prueba Chi Cuadrado de Pearson

\begin{tabular}{|c|c|c|c|}
\hline & Valor & gl & Significancia \\
\hline Chi Cuadrado de Pearson & $91.704^{\mathrm{a}}$ & 15 & .000 \\
\hline Razón de verosimilitudes & 64.471 & 15 & .000 \\
\hline Asociación lineal por lineal & 28.313 & 1 & .000 \\
\hline N de casos válidos & 150 & & \\
\hline
\end{tabular}

Elaborado por: Equipo de investigación, 2016.

Para que la relación entre las variables se pueda explicar el p-valor arrojado por la prueba debe ser inferior al 5\%. En el caso de la investigación, el p-valor arrojado es del 5\%, lo que demuestra que existe una relación directa entre las variables. De acuerdo a los resultados de la tabla de contingencia se puede concluir que mientras mayor sea el grado de aplicación del método de BATHE, mayor es el grado de satisfacción de los padres en la consulta preoperatoria de sus hijos.

\section{Conclusión}

En lo que respecta a las preguntas más sobresalientes del método BATHE se tiene que el porcentaje de satisfacción de los padres de los pacientes que acuden a la consulta preanestésica fue "buena" en el 57,33\% y "muy buena" en el 24,67\%. La diferencia entre resultados obedece a varios factores; el primero es que el tiempo de la consulta es limitado, motivo por el cual en la consulta preanestésica se da énfasis a los aspectos más relevantes desde el punto de vista técnico; es decir, al examen físico y a los resultados de laboratorio e imagen. Otro factor es que la consulta no está sistematizada, por lo tanto el médico no siempre sigue un orden lógico y la entrevista se desarrolla conforme a la situación de cada paciente. Por último se encuentran los modelos obsoletos de atención médica no socializada, pero si gerenciada, descritos por Ceriani J, (2011) donde la prioridad es la atención médica en base a producción, lo cual disminuye la motivación y la satisfacción de ejercer 
la profesión médica.

Al 74,7\% de padres encuestados, el médico les interrogó sobre cuáles son los temores con respecto a la cirugía de sus hijos y a menos de la mitad (42\% de padres), se les indagó acerca de la manera en que ellos están manejando esos temores, y cómo los pacientes que van a ser sometidos a cualquier tipo de intervención quirúrgica presentan sensaciones inherentes al ser humano, como: el miedo, la ansiedad, el temor a lo desconocido, a las complicaciones posteriores a la cirugía y al dolor. Se ha comprobado que todos estos temores pueden reducirse con una mayor información por parte del médico en la consulta de cirugía y preanestésica.

El nivel de seguridad percibido por los padres de los pacientes prequirúrgicos, que acuden a la consulta preanestésica pediátrica es alto; con un nivel de confianza del 73,33\% entre "bueno" y "muy bueno", y un nivel de tranquilidad respecto a sus dudas y ansiedades de $57,33 \%$ considerado como "bueno", dando como resultado que después de concluida la visita preanestésica los padres están seguros de que sus hijos se recuperarán de manera oportuna y positiva.

Estos resultados responden a la importancia sobre el conocimiento, manejo y en la aplicación adecuada del método de BATHE en las futuras consultas preanestésicas. Dicho método puede ser aplicado y adaptado a las características propias de cada uno de los hospitales de segundo y tercer nivel de atención de salud en el Ecuador, determinando que la utilización de este método hará que el servicio de atención al paciente en la consulta sea más óptimo, dejando a un lado cualquier duda o inquietud tanto del paciente como de los familiares.

\section{References:}

1. Ceriani, J. (2011). La insatisfacción en la práctica médica. IntraMed. Recuperado de: https://www.intramed.net/contenidover.asp?contenidoID=73802

2. DeMaria, S. J., DeMaria, A. P., Silvay, G., \& Flynn, B. (2011). Use of the BATHE Method in the Preanesthetic Clinic Visit. International Anesthesia Research Society, $X(\mathrm{X})$.

3. Gómez, J. G., Vinagre, J. C., Hita, E. O., \& Macía (2008). Nuevas metodologías en el entrenamiento de emergencias pediátricas: simulación médica aplicada a pediatría. In Anales de Pediatría, (Vol. 68, No. 6, pp. 612-620). Elsevier Doyma.

4. Liondas, S. (2009). Evaluación y desarrollo de la anestesiología. . Anestesia Analgesia Reanimación,, 22(1), 5-18.

5. Mira , J., \& Aranaz , J. (2000). La satisfacción del paciente como una medidad del resultado de la atención sanitaria. Medicina Clínica., 114(3): p. 5. 
6. Pitarch, J. L., \& Ibáñez, A. (2005). Guía clínica de fármacos inhibidores de la hemostasia y anestesia regional neuroaxial. Revista Española Anestesiol., 52(7).

7. Rivera F, J. (2004). Aspectos legales en anestesia para cirugía de urgencia. Revista Mexicana de Anestesiología, 27(S1), 185-187.

\section{Anexo Encuesta}

\section{PRIMERA PARTE}

Por favor responda sí o no las siguientes preguntas (marque con un círculo en la respuesta):

1. El médico le preguntó a usted, ¿cuál es el motivo de su visita o que lo trae por aquí hoy? SI NO

2. El médico le preguntó a usted, ¿cómo le está afectando la idea de operar a su hijo/a? SI NO

3. El médico le preguntó a usted, ¿cuáles son sus temores respecto a la cirugía? SI NO

4. El médico le preguntó a usted, ¿cómo está manejando estas preocupaciones? SI NO

5. ¿El médico, fue comprensivo con sus preocupaciones?

SI NO

SEGUNDA PARTE

Por favor responda las preguntas de la 6 a la 20 usando la siguiente escala (Seleccione la casilla)

○ Muy pobre

- Pobre

- Justa (normal)

○ Buena

- Muy buena

\begin{tabular}{llllll}
\hline 6. Amabilidad de su médico & Muy pobre & Pobre & Justa & Buena & Muy buena \\
\hline $\begin{array}{l}\text { 7. Explicación del médico sobre las } \\
\text { preguntas que usted tuvo. }\end{array}$ & Muy pobre & Pobre & Justa & Buena & Muy buena \\
\hline $\begin{array}{l}\text { 8. Preocupación del médico sobre sus } \\
\text { miedos. }\end{array}$ & Muy pobre & Pobre & Justa & Buena & Muy buena \\
\hline $\begin{array}{l}\text { 9. Esfuerzo del médico por incluirle en } \\
\text { las decisiones sobre la anestesia. }\end{array}$ & Muy pobre & Pobre & Justa & Buena & Muy buena \\
\hline $\begin{array}{l}\text { 10. Información sobre el tipo de } \\
\text { anestesia. }\end{array}$ & Muy pobre & Pobre & Justa & Buena & Muy buena \\
\hline $\begin{array}{l}\text { 11. Instrucciones sobre el seguimiento y } \\
\text { recuperación de la anestesia. }\end{array}$ & Muy pobre & Pobre & Justa & Buena & Muy buena \\
\hline
\end{tabular}




\begin{tabular}{lllllll}
\hline 12. Uso de palabras comprensibles por & Muy pobre & Pobre & Justa & Buena & Muy buena \\
parte del médico. & & & & & \\
\hline $\begin{array}{l}\text { 13. Cantidad de tiempo que el médico } \\
\text { pasó con usted. }\end{array}$ & Muy pobre & Pobre & Justa & Buena & Muy buena \\
$\begin{array}{l}\text { 14. Nivel de confianza sobre el médico } \\
\text { que les atendió. }\end{array}$ & Muy pobre & Pobre & Justa & Buena & Muy buena \\
\hline $\begin{array}{l}\text { 15. Recomendación del médico para otras } \\
\text { personas. }\end{array}$ & Muy pobre & Pobre & Justa & Buena & Muy buena \\
\hline $\begin{array}{l}\text { 16. Percepción del cuidado que recibirá } \\
\text { por parte del anestesiólogo. }\end{array}$ & Muy pobre & Pobre & Justa & Buena & Muy buena \\
$\begin{array}{l}\text { 17. ¿Cree que su hijo/a está preparado } \\
\text { para la recuperación después de la } \\
\text { cirugía, basado en la consulta } \\
\text { preanestésica? }\end{array}$ & & Pobre & Justa & Buena & Muy buena \\
\hline $\begin{array}{l}\text { 18. Opinión personal del médico que les } \\
\text { atendió. }\end{array}$ & Muy pobre & Pobre & Justa & Buena & Muy buena \\
\hline $\begin{array}{l}\text { 19. ¿Qué tanto alivio le brindó la consulta } \\
\text { preanestésica sobre sus dudas y la } \\
\text { ansiedad por la cirugía? }\end{array}$ & & Puy pobre & Pobre & Justa & Buena & Muy buena \\
\hline $\begin{array}{l}\text { 20. Satisfacción general posterior a la } \\
\text { consulta preanestésica. }\end{array}$ & Muy pobre & Pobre & Justa & Buena & Muy buena \\
\hline
\end{tabular}

